\title{
Loop corrections in double field theory: non-trivial dilaton potentials
}

\author{
Songlin Lv, ${ }^{a}$ Houwen $\mathbf{W} \mathbf{u}^{a}$ and Haitang Yang ${ }^{a, b}$ \\ ${ }^{a}$ Center for theoretical physics, College of Physical Science and Technology, Sichuan University, \\ Chengdu 610064, P.R. China \\ ${ }^{b}$ Kavli Institute for Theoretical Physics China (KITPC), Chinese Academy of Sciences, \\ Beijing 100080, P.R. China \\ E-mail: 2013222020005@stu.scu.edu.cn, 2013222020003@stu.scu.edu.cn, \\ hyanga@scu.edu.cn
}

ABSTRACT: It is believed that the invariance of the generalised diffeomorphisms prevents any non-trivial dilaton potential from double field theory. It is therefore difficult to include loop corrections in the formalism. We show that by redefining a non-local dilaton field, under strong constraint which is necessary to preserve the gauge invariance of double field theory, the theory does permit non-constant dilaton potentials and loop corrections. If the fields have dependence on only one single coordinate, the non-local dilaton is identical to the ordinary one with an additive constant.

KEYwords: String Duality, String Field Theory

ARXIV EPRINT: 1408.3713 


\section{Contents}

1 Introduction 1

2 Generalized Lie derivatives and gauge scalar 3

3 Non-local dilaton potential in DFT 5

4 Conclusion $\quad 8$

\section{Introduction}

Born from closed string field theory, double field theory (DFT) [1-11] is constructed by formally doubling all spacetime coordinates of the massless sector of closed string spectrum. DFT manifests an $O(D, D)$ symmetry explicitly and the dual coordinates represent the conjugation of winding numbers. Formally, all components of closed string fields are formulated by double coordinates $\phi_{I}\left(X^{M}\right)$, where $X^{M}=\left(\tilde{x}_{i}, x^{i}\right), M=1,2, \ldots, 2 D$ and $i=1,2, \ldots, D$. In such a formalism, $x^{i}$ is the usual coordinates and $\tilde{x}_{i}$ denotes the dual coordinates of winding momentum. Good reviews of DFT are given by [12-15].

To build an $O(D, D)$ invariant spacetime action, a generalized $O(D, D)$ metric is introduced

$$
\mathcal{H}_{M N}=\left(\begin{array}{cc}
g^{i j} & -g^{i k} b_{k j} \\
b_{i k} g^{k j} & g_{i j}-b_{i k} g^{k l} b_{l j}
\end{array}\right),
$$

unifying the spacetime metric $g_{i j}$ and anti-symmetric Kalb-Ramond field $b_{i j}$ altogether. In this metric, $M$ is an $O(D, D)$ index, running from 1 to $2 D$. The $O(D, D)$ invariant spacetime action is built by contraction of $O(D, D)$ indices,

$$
\begin{gathered}
S=\int d^{D} x d^{D} \tilde{x} e^{-2 d}\left(\frac{1}{8} \mathcal{H}^{M N} \partial_{M} \mathcal{H}^{K L} \partial_{N} \mathcal{H}_{K L}-\frac{1}{2} \mathcal{H}^{M N} \partial_{N} \mathcal{H}^{K L} \partial_{L} \mathcal{H}_{M K}\right. \\
\left.-\partial_{M} d \partial_{N} \mathcal{H}^{M N}+4 \mathcal{H}^{M N} \partial_{M} d \partial_{N} d\right)
\end{gathered}
$$

where $d$ is an $O(D, D)$ scalar dilaton, defined by the usual dilaton $\phi$ through $e^{-2 d}=$ $\sqrt{g} e^{-2 \phi}$. Therefore, DFT is an effective theory for three massless fields: $D$ dimensional spacetime metric $g_{i j}$, the anti-symmetric Kalb-Ramond field $b_{i j}$ and the scalar dilaton $\phi$. If we compactify $d$ dimensions of $D=n+d$, the continuous $O(D, D)$ group breaks to $O(n, n) \times O(d, d ; Z)$, where $O(n, n)$ is still a continuous group and $O(d, d ; Z)$ is T-duality in the compactified background. To be a consistent theory, DFT is required to be invariant under the gauge transformations

$$
\delta_{\xi} \mathcal{H}^{M N}=\hat{\mathcal{L}}_{\xi} \mathcal{H}^{M N} \equiv \xi^{P} \partial_{P} \mathcal{H}^{M N}+\left(\partial^{M} \xi_{P}-\partial_{P} \xi^{M}\right) \mathcal{H}^{P N}+\left(\partial^{N} \xi_{P}-\partial_{P} \xi^{N}\right) \mathcal{H}^{M P},
$$




$$
\delta d=\xi^{M} \partial_{M} d-\frac{1}{2} \partial_{M} \xi^{M}
$$

where $\xi^{M}=\left(\tilde{\xi}_{i}, \xi^{i}\right)$ and $\hat{\mathcal{L}}_{\xi}$ is the "generalized Lie derivatives". Since DFT is based on closed string theory, it must satisfy the level matching condition: $L_{0}-\bar{L}_{0}=-p_{i} w^{i}=0$ for massless states. In the language of DFT, the level matching condition is transformed to the weak constraint: $\partial^{M} \partial_{M} A=0$ where $\partial_{M}=\left(\tilde{\partial}^{i}, \partial_{i}\right)$ and $A$ stands for an arbitrary field. However, the weak constraint is insufficient to guarantee the gauge invariance. In order to make $\tilde{\partial}^{i} \partial_{i}\left(\delta_{\xi} \Phi\right)=\tilde{\partial}^{i} \partial_{i}(\xi \cdot \Phi)=0$, a much stronger constraint is imposed: $\partial^{M} \partial_{M}(\cdot)$ where - denotes any product of fields or gauge parameters. Under this strong constraint, only half of the coordinates survive for all fields and gauge parameters and DFT reduces to the $D$ dimensional traditional low energy effective theory. In addition, there are many works to imply that the strong constraint can be relaxed on a torus background, massive type IIA and gauged supergravity [16-20]. The detailed discussions on constraint relaxation are summarized in $[15,21]$.

It is widely believed that a non-trivial dilaton potential is forbidden by the generalised diffeomorphism in DFT [22-25]. Considering the DFT action $S=\int d x d \tilde{x} e^{-2 d} \mathcal{R}$, it proves that $\mathcal{R}$ is an $O(D, D)$ scalar and also a gauge scalar. Moreover, since the weight of the $O(D, D)$ scalar $e^{-2 d}$ equals the unity, it is a density which is invariant under the generalised diffeomorphisms when combined with the proper volume $\int d x d \tilde{x}$. The point is that $\int d x d \tilde{x} e^{-2 d}$ is the unique multiplying factor of the dilaton that respects the generalised diffeomorphisms and $O(D, D)$ symmetry. This is such a strong constraint that higher loop corrections are completely excluded! It is worth noting that the dilaton will always increase as time goes by and it also marks the growth of the curvature. However, the growth of the string coupling $g_{s}=\exp (2 \phi)$ and the growth of the Hubble parameter $H$ lead the universe approaching two limits [26], or two corrections to the low energy effective action: (1) the string curvature scale, which requires the $\alpha^{\prime}$ corrections to the low energy effective action when $\sqrt{2 \pi \alpha^{\prime}} H$ reaches 1 , and (2) the strong coupling regime, which requires the quantum loop corrections of the form $e^{2 n \phi}(\ldots)$ for non-negative integer $n$, when $g_{s} \sim 1$. The first expansion has been discussed in ref. [27-30]. It would be unnatural that loop corrections totally disappear in the formalism, since beyond both limits, the universe enters the string non-perturbative regime, and the action with these two corrections will give us some non-perturbative signatures which should be described by the yet-to-know M-theory.

The aim of this paper is to address the higher loop quantum corrections in DFT. To achieve this purpose, one does not really need to consider the complete loop expansion but justification of a pure dilaton potential is sufficient. However, a simple dilaton potential of the form $V\left(e^{d}\right)$ does not work, since the product $e^{-2 d} V\left(e^{d}\right)$ is no longer a density and breaks the gauge invariance of the action, though it is an $O(D, D)$ scalar. It turns out that in order to preserve the symmetries, a non-local dilaton $d_{*}$ has to be defined to replace the ordinary local dilaton by the similar method used in the traditional string cosmology [31]. Since this non-local dilaton $e^{-2 d_{*}}$ includes a proper volume, it does not break the gauge invariance under the strong constraint. Then additional potential terms of DFT action could be any regular function of this non-local dilaton. Moreover, if we choose 
the cosmological background and the cosmic-time gauge, the non-local dilaton $e^{-2 d_{*}}$ reduces to the usual $O(D, D)$ scalar dilaton $e^{-2 d}$ multiplied by a proper volume. The cosmological implications with this non-local dilaton have been discussed in our previous works [32, 33]. Ref. [34] discussed cosmological solutions with a constant dilaton potential.

The reminder of this paper is outlined as follows. In section 2, we discuss the gauge transformations of DFT. We define the non-local dilaton in section 4 . Section 5 is our conclusion and discussions.

\section{Generalized Lie derivatives and gauge scalar}

To begin with, we give a brief review of the gauge transformations based on refs. [4-7]. The DFT action, expanded in terms of $g_{i j}, b_{i j}$ and $d$, can be recasted as

$$
S=S^{(0)}+S^{(1)}+S^{(2)}
$$

with

$$
S^{(k)}=\int d x d \tilde{x} \mathcal{L}^{(k)}, \quad k=0,1,2
$$

where the superscript denotes the number of $\tilde{\partial}$ in the DFT action. The full gauge transformations can be written as

$$
\begin{aligned}
\delta_{\xi} g_{i j} & =\mathcal{L}_{\xi} g_{i j}+\mathcal{L}_{\tilde{\xi}} g_{i j}+2\left(\tilde{\partial}^{k} \xi^{l}-\tilde{\partial}^{l} \xi^{k}\right)\left(g_{k i} b_{j l}+g_{k j} b_{i l}\right), \\
\delta_{\xi} g^{i j} & =\mathcal{L}_{\xi} g^{i j}+\mathcal{L}_{\tilde{\xi}} g^{i j}-\left[\left(\tilde{\partial}^{i} \xi^{k}-\tilde{\partial}^{k} \xi^{i}\right) g^{j l} b_{l k}+(i \leftrightarrow j)\right], \\
\delta_{\xi} b_{i j} & =\mathcal{L}_{\xi} b_{i j}+\mathcal{L}_{\tilde{\xi}} b_{i j}+\partial_{i} \tilde{\xi}_{j}-\partial_{j} \tilde{\xi}_{i}+g_{i k}\left(\tilde{\partial}^{l} \xi^{k}-\tilde{\partial}^{k} \xi^{l}\right) g_{l j}+b_{i k}\left(\tilde{\partial}^{l} \xi^{k}-\tilde{\partial}^{k} \xi^{l}\right) b_{l j}, \\
\delta_{\xi} d & =\left(\xi^{i} \partial_{i}+\tilde{\xi}_{i} \tilde{\partial}^{i}\right) d-\frac{1}{2}\left(\partial_{i} \xi^{i}+\tilde{\partial}^{i} \tilde{\xi}_{i}\right),
\end{aligned}
$$

where $\mathcal{L}_{\xi}$ is the Lie derivatives with respect to $\xi, \mathcal{L}_{\tilde{\xi}}$ is the dual Lie derivatives with respect to $\tilde{\xi}, \xi$ and $\tilde{\xi}$ are gauge parameters. The Lie derivatives and its dual for arbitrary tensors $u_{i}^{j}$ can be defined as follows

$$
\begin{aligned}
& \mathcal{L}_{\xi} u_{i}^{j}=\xi^{p} \partial_{p} u_{i}^{j}+\partial_{i} \xi^{p} u_{p}^{j}+\partial_{p} \xi^{j} u_{i}^{p}, \\
& \mathcal{L}_{\tilde{\xi}} u_{i}^{j}=\tilde{\xi}_{p} \tilde{\partial}^{p} u_{i}^{j}-\tilde{\partial}^{j} \tilde{\xi}_{p} u_{i}^{p}-\tilde{\partial}^{p} \tilde{\xi}_{i} u_{p}^{j} .
\end{aligned}
$$

We can split it into two parts according to the number of $\tilde{\partial}$ acted

$$
\delta_{\xi}=\delta_{\xi}^{(0)}+\delta_{\xi}^{(1)}
$$

These gauge transformations $\delta_{\xi}^{(0)}$ and $\delta_{\xi}^{(1)}$ are T-dual with each other. For example, to consider the gauge transformations for dilaton $\delta_{\xi} d$, we have

$$
\begin{aligned}
& \delta_{\xi}^{(0)} d=\xi^{i} \partial_{i} d-\frac{1}{2} \partial_{i} \xi^{i}, \\
& \delta_{\xi}^{(1)} d=\tilde{\xi}_{i} \tilde{\partial}^{i} d-\frac{1}{2} \tilde{\partial}^{i} \tilde{\xi}_{i} .
\end{aligned}
$$


In order to check the gauge invariance, we need to prove that

$$
\left(\delta_{\xi}^{(0)}+\delta_{\xi}^{(1)}\right)\left(S^{(0)}+S^{(1)}+S^{(2)}\right)=0 .
$$

In other words, this equation requires the following conditions

$$
\begin{aligned}
\delta_{\xi}^{(0)} S^{(0)} & =0, \\
\delta_{\xi}^{(1)} S^{(2)} & =0, \\
\delta_{\xi}^{(0)} S^{(1)}+\delta_{\xi}^{(1)} S^{(0)} & =0, \\
\delta_{\xi}^{(1)} S^{(1)}+\delta_{\xi}^{(0)} S^{(2)} & =0 .
\end{aligned}
$$

Since equations (2.8) and (2.10) are T-dual versions of equations (2.9) and (2.11) respectively, we only need to check equations (2.8) and (2.10). It is easy to see that equation (2.8) is automatically satisfied since it is the standard gauge transformations of Einstein's gravity. One only needs to verify equation (2.10). Furthermore, since the Lie derivative terms can be combined into total derivatives, one does not need to consider them in the calculation. Because of the independence of gauge parameters $\xi_{i}$ and $\tilde{\xi}_{i}$, we can check the gauge invariance with each of them respectively. For example, to check gauge invariance of equation $(2.10)$, we can set $\tilde{\xi}_{i}=0$ and $\xi_{i} \neq 0$, and vice versa.

In the language of $O(D, D)$ symmetry, all gauge transformations above can be rewritten in terms of $O(D, D)$ indices. For a tensor $A_{M}{ }^{N}$, the generalized Lie derivative is defined as

$$
\hat{\mathcal{L}}_{\xi} A_{M}{ }^{N} \equiv \xi^{P} \partial_{P} A_{M}{ }^{N}+\left(\partial_{M} \xi^{P}-\partial^{P} \xi_{M}\right) A_{P}{ }^{N}+\left(\partial^{N} \xi_{P}-\partial_{P} \xi^{N}\right) A_{M}{ }^{P}
$$

The generalized Lie derivative also satisfies the Leibniz rule. The gauge transformation for the generalized metric is

$$
\delta_{\xi} \mathcal{H}^{M N}=\hat{\mathcal{L}}_{\xi} \mathcal{H}^{M N} .
$$

For a scalar $S$ or a generalized scalar $A_{M}{ }^{M}$, the generalized Lie derivative is simply

$$
\hat{\mathcal{L}}_{\xi} S=\xi^{P} \partial_{P} S, \quad \hat{\mathcal{L}}_{\xi} A_{M}^{M}=\xi^{P} \partial_{P} A_{M}{ }^{M} .
$$

To consider the gauge transformations of any object $W$, we can split it into two parts

$$
\delta_{\xi} W=\hat{\mathcal{L}}_{\xi} W+\triangle_{\xi} W,
$$

where $\triangle_{\xi}$ also satisfies the Leibniz rule: $\triangle_{\xi}(W V)=\left(\triangle_{\xi} W\right) V+W\left(\triangle_{\xi} V\right)$. The first term of (2.15) is a Lie derivative, therefore it is covariant and we do not need to consider it as we explained above. The second term of (2.15) transforms as a tensor. Therefore, it suffices to check $\triangle_{\xi} W=0$ to confirm the gauge invariance of the DFT action. Now, recall the DFT action,

$$
S=\int d x d \tilde{x} e^{-2 d} \mathcal{R},
$$


where

$$
\begin{aligned}
\mathcal{R}= & \frac{1}{8} \mathcal{H}^{M N} \partial_{M} \mathcal{H}^{K L} \partial_{N} \mathcal{H}_{K L}-\frac{1}{2} \mathcal{H}^{M N} \partial_{N} \mathcal{H}^{K L} \partial_{L} \mathcal{H}_{M K} \\
& -\partial_{M} d \partial_{N} \mathcal{H}^{M N}+4 \mathcal{H}^{M N} \partial_{M} d \partial_{N} d .
\end{aligned}
$$

We can find that

$$
\delta_{\xi} \mathcal{R}=\hat{\mathcal{L}}_{\xi} \mathcal{R}=\xi^{M} \partial_{M} \mathcal{R},
$$

with $\triangle_{\xi} \mathcal{R}=0$. Therefore, $\mathcal{R}$ is a gauge scalar. Moreover, the dilaton term gives

$$
\delta_{\xi} e^{-2 d}=\hat{\mathcal{L}}_{\xi} e^{-2 d}=\partial_{M}\left(\xi^{M} e^{-2 d}\right),
$$

where $\hat{\mathcal{L}}_{\xi} e^{-2 d}=-2\left(\hat{\mathcal{L}}_{\xi} d\right) e^{-2 d}$ and $\hat{\mathcal{L}}_{\xi} d=\xi^{M} \partial_{M} d-\frac{1}{2} \partial_{M} \xi^{M}$. Since, the wight of this term equals the unity, it is a scalar density. To calculate the weight of a density, we can use the method introduced in refs. [35-38]. We first introduce the semi-covariant derivative, $\nabla_{C}=\partial_{C}+\Gamma_{C}:$

$$
\nabla_{C} T_{\omega A_{1} A_{2} \cdots A_{n}}=\partial_{C} T_{\omega A_{1} A_{2} \cdots A_{n}}-\omega \Gamma^{B}{ }_{B C} T_{\omega A_{1} A_{2} \cdots A_{n}}+\sum_{i=1}^{n} \Gamma_{C A_{i}}{ }^{B} T_{\omega A_{1} \cdots A_{i-1} B A_{i+1} \cdots A_{n}}
$$

where $T_{\omega A_{1} A_{2} \cdots A_{n}}$ is a field and $\omega$ is the weight to identify each field. For example, considering the dilaton term $e^{-2 d}$, it is easy to find

$$
\nabla_{C} e^{-2 d}=\left(-2 \nabla_{C} d\right) e^{-2 d}=\partial_{C} e^{-2 d}-\Gamma_{B C}^{B} e^{-2 d},
$$

where $\nabla_{C} d=\partial_{C} d+\frac{1}{2} \Gamma_{B C}^{B}$. It implies that the dilaton potential $e^{-2 d}$ has a weight $\omega=1$.

In summary, the action is gauge invariant under the strong constraint. However, if we introduce a dilaton potential, say, $V(d)=e^{8 d}$, we will get a term

$$
\int d x d \tilde{x} e^{-2 d} V(d)=\int d x d \tilde{x} e^{6 d} .
$$

The weight of this term is not the unity, thus it is not a scalar density and breaks the gauge invariance. In the next section, we will solve this problem by redefining the dilaton, a generalisation of the results in the traditional string cosmology [31].

\section{Non-local dilaton potential in DFT}

We would like to emphasize again that the strong constraint $\partial^{M} \partial_{M}(\cdot)$ is necessary to make the DFT action (2.16) gauge invariant. Therefore, we are not trying to construct a gauge invariant potential without imposing the strong constraint, though it must be $O(D, D)$ invariant at the first place. We define a non-local $O(D, D)$ invariant dilaton $d_{*}(x, \tilde{x})$ as

$$
\begin{aligned}
e^{-2 d_{*}(x, \tilde{x})} \equiv & \int d^{D} x^{\prime} d^{D} \tilde{x}^{\prime} e^{-2 d\left(x^{\prime}, \tilde{x}^{\prime}\right)}\left[2 \sqrt{-\partial_{\mu} \phi\left(x^{\prime}, \tilde{x}^{\prime}\right) \partial^{\mu} \phi\left(x^{\prime}, \tilde{x}^{\prime}\right)} \delta\left(\phi(x, \tilde{x})-\phi\left(x^{\prime}, \tilde{x}^{\prime}\right)\right)\right. \\
& \left.+2 \sqrt{-\tilde{\partial}_{\mu} \tilde{\phi}\left(x^{\prime}, \tilde{x}^{\prime}\right) \tilde{\partial} \mu \tilde{\phi}\left(x^{\prime}, \tilde{x}^{\prime}\right) \delta}\left(\tilde{\phi}(x, \tilde{x})-\tilde{\phi}\left(x^{\prime}, \tilde{x}^{\prime}\right)\right)\right]
\end{aligned}
$$


where $\tilde{g}_{\mu \nu}=g^{\mu \nu}, d\left(x^{\prime}, \tilde{x}^{\prime}\right)=\tilde{\phi}+\frac{1}{4} \ln (-g)=\phi-\frac{1}{4} \ln (-g)$ to preserve the $O(D, D)$ symmetry. To check the gauge invariance of this non-local dilaton $d_{*}(x, \tilde{x})$ under the strong constraint, we first write it in the form

$$
d_{*}(x, \tilde{x})=\mathcal{V}_{*}^{(0)}(x, \tilde{x})+\mathcal{V}_{*}^{(2)}(x, \tilde{x}),
$$

where the superscript is the number of $\tilde{\partial}$ derivatives and $\mathcal{V}_{*}^{(0)}, \mathcal{V}_{*}^{(2)}$ are T-dual with each other,

$$
\begin{aligned}
& \mathcal{V}_{*}^{(0)}(x, \tilde{x}) \equiv \int d^{D} x^{\prime} d^{D} \tilde{x}^{\prime} e^{-2 d\left(x^{\prime}, \tilde{x}^{\prime}\right)} 2 \sqrt{-\partial_{\mu} \phi\left(x^{\prime}, \tilde{x}^{\prime}\right) \partial^{\mu} \phi\left(x^{\prime}, \tilde{x}^{\prime}\right)} \delta\left(\phi(x, \tilde{x})-\phi\left(x^{\prime}, \tilde{x}^{\prime}\right)\right), \\
& \mathcal{V}_{*}^{(2)}(x, \tilde{x}) \equiv \int d^{D} x^{\prime} d^{D} \tilde{x}^{\prime} e^{-2 d\left(x^{\prime}, \tilde{x}^{\prime}\right)} 2 \sqrt{-\tilde{\partial}_{\mu} \tilde{\phi}\left(x^{\prime}, \tilde{x}^{\prime}\right) \tilde{\partial}^{\mu} \tilde{\phi}\left(x^{\prime}, \tilde{x}^{\prime}\right)} \delta\left(\tilde{\phi}(x, \tilde{x})-\tilde{\phi}\left(x^{\prime}, \tilde{x}^{\prime}\right)\right) .
\end{aligned}
$$

It has no harm to include a $\mathcal{V}_{*}^{(1)}$ term in the definition (3.1), nevertheless it will be killed by the strong constraint. To respect the gauge symmetry, under the strong constraint, equation (3.2) must satisfy

$$
\left(\delta_{\xi}^{(0)}+\delta_{\xi}^{(1)}\right)\left(\mathcal{V}_{*}^{(0)}+\mathcal{V}_{*}^{(2)}\right)=0
$$

We know that $\delta_{\xi}^{(0)} \mathcal{V}_{*}^{(0)}=0$ is the standard gauge invariance of the traditional string cosmology, and $\delta_{\xi}^{(1)} \mathcal{V}_{*}^{(2)}=0$ is the T-dual version of it. Since $\delta_{\xi}^{(0)} \mathcal{V}_{*}^{(2)}$ is the T-dual of $\delta_{\xi}^{(1)} \mathcal{V}_{*}^{(0)}$, we only need to check $\delta_{\xi}^{(1)} \mathcal{V}_{*}^{(0)}=0$ by setting $\xi_{i}$ non-zero or $\tilde{\xi}_{i}$ non-zero respectively. Bear in mind that imposing the strong constraint is equivalent to setting all fields having dependence on only half of the doubled coordinates. Even the first term in equation (3.1), the original DFT action, is not gauge invariant without imposing the strong constraint. Therefore, when check the gauge invariance of $d_{*}(x, \tilde{x})$, we can assume all field to depend on only one set of coordinates. Looking back at equation (3.3), it implies that to get a nonvanishing $\mathcal{V}_{*}^{(0)}$, we have $\tilde{\partial} \cdot A=0$ for an arbitrary field or parameter $A$.

When $\tilde{\xi}_{i}$ is non-zero, we have

$$
\delta^{(1)} g_{i j}=\mathcal{L}_{\tilde{\xi}} g_{i j}, \quad \delta^{(1)} b_{i j}=\mathcal{L}_{\tilde{\xi}} b_{i j}, \quad \delta^{(1)} d=\tilde{\xi}_{i} \tilde{\partial}^{i} d-\frac{1}{2} \tilde{\partial}^{i} \tilde{\xi}_{i}
$$

We thus obtain

$$
\begin{aligned}
\delta^{(1)} \mathcal{V}_{*}^{(0)}= & \int d^{D} x^{\prime} d^{D} \tilde{x}^{\prime} \tilde{\partial}^{i}\left(\tilde{\xi}_{i} e^{-2 d}\right) 2 \sqrt{-\partial_{\mu} \phi\left(x^{\prime}, \tilde{x}^{\prime}\right) \partial^{\mu} \phi\left(x^{\prime}, \tilde{x}^{\prime}\right)} \delta\left(\phi(x, \tilde{x})-\phi\left(x^{\prime}, \tilde{x}^{\prime}\right)\right) \\
& +\int d^{D} x^{\prime} d^{D} \tilde{x}^{\prime} e^{-2 d\left(x^{\prime}, \tilde{x}^{\prime}\right)} 2\left(\delta^{(1)} \sqrt{-\partial_{\mu} \phi\left(x^{\prime}, \tilde{x}^{\prime}\right) \partial^{\mu} \phi\left(x^{\prime}, \tilde{x}^{\prime}\right)}\right) \delta\left(\phi(x, \tilde{x})-\phi\left(x^{\prime}, \tilde{x}^{\prime}\right)\right),
\end{aligned}
$$

where we used $\delta^{(1)} e^{-2 d}=\tilde{\partial}^{i}\left(\tilde{\xi}_{i} e^{-2 d}\right)$ to get the first term on the r.h.s. Since under the strong constraint, $\delta^{(1)} \phi=\tilde{\xi}_{i} \tilde{\partial}^{i} \phi+\tilde{\partial}^{i} \tilde{\xi}_{i}=0$, the second term on the r.h.s. vanishes and the first term is a total derivative. Therefore, we have $\delta^{(1)} \mathcal{V}_{*}^{(0)}=0$. On the other hand, when $\xi_{i}$ is non-zero, the gauge variations are

$$
\delta^{(1)} g_{i j}=2\left(\tilde{\partial}^{k} \xi^{l}-\tilde{\partial}^{l} \xi^{k}\right) g_{k(i} b_{j) l}
$$




$$
\begin{aligned}
& \delta^{(1)} g^{i j}=-\left(\tilde{\partial}^{i} \xi^{k}-\tilde{\partial}^{k} \xi^{i}\right) g^{j l} b_{l k}+(i \leftrightarrow j), \\
& \delta^{(1)} b_{i j}=g_{i k}\left(\tilde{\partial}^{l} \xi^{k}-\tilde{\partial}^{k} \xi^{l}\right) g_{l j}+b_{i k}\left(\tilde{\partial}^{l} \xi^{k}-\tilde{\partial}^{k} \xi^{l}\right) b_{l j},
\end{aligned}
$$

and we have

$$
\delta^{(1)} \mathcal{V}_{*}^{(0)}=\delta^{(1)} \int d^{D} x^{\prime} d^{D} \tilde{x}^{\prime} e^{-2 d\left(x^{\prime}, \tilde{x}^{\prime}\right)} 2 \sqrt{-\partial_{\mu} \phi\left(x^{\prime}, \tilde{x}^{\prime}\right) \partial^{\mu} \phi\left(x^{\prime}, \tilde{x}^{\prime}\right)} \delta\left(\phi(x, \tilde{x})-\phi\left(x^{\prime}, \tilde{x}^{\prime}\right)\right)
$$

Since $\tilde{\xi}_{i}=0$, we have $\delta^{(1)} d=\tilde{\xi}_{i} \tilde{\partial}^{i} d-\frac{1}{2} \tilde{\partial}^{i} \tilde{\xi}_{i}=0$. After applying the strong constraint, it is easy to see $\delta^{(1)} \phi=\tilde{\xi}_{i} \tilde{\partial}^{i} \phi+\tilde{\partial}^{i} \tilde{\xi}_{i}=0$. We thus conclude $\delta^{(1)} \mathcal{V}_{*}^{(0)}=0$ for both cases and then $d_{*}(x, \tilde{x})$ is a gauge scalar under the strong constraint. Moreover, since the definition of the non-local dilaton (3.1) is independent of $b$-field, we do not need to consider the C-bracket, ${ }^{1}$ and the closure of the Lie algebra is preserved [4-7].

Given $d_{*}(x, \tilde{x})$ is a gauge scalar, phenomenologically, any regular function of $d_{*}(x, \tilde{x})$ could serve as a non-trivial dilaton potential in the DFT action

$$
S=\int d x d \tilde{x} e^{-2 d}\left[\mathcal{R}-V\left(d_{*}(x, \tilde{x})\right)\right] .
$$

Nevertheless, it is possible to derive a more realistic dilaton potential from loop corrections. To fulfill this purpose, one needs first to show that in DFT, the $n$-th loop correction $S_{n}$ can be organized in the form

$$
S_{n}=\int d x d \tilde{x} e^{-2 d} e^{2 n d_{*}}(\cdots), \quad n \geq 1,
$$

where $(\cdots)$ denotes gauge and $O(D, D)$ scalars. Then, loop by loop, dilaton potentials can be obtained by solving the equations of motion.

We claim that if the fields have only one single coordinate dependence, the non-local dilaton (3.1) reduces to the ordinary one. Let us choose the cosmological background with the cosmic-time gauge $\left(g_{00}=-1\right)$, the non-local dilaton (3.1) becomes

$$
d_{*}(t)=-\frac{1}{2} \ln V_{d} \int d \phi\left(t^{\prime}\right) \sqrt{-g\left(t^{\prime}\right)} e^{-2 \phi\left(t^{\prime}\right)} \delta\left(\phi(t)-\phi\left(t^{\prime}\right)\right)=d(t)-\frac{1}{2} \ln V_{d},
$$

or

$$
d_{*}(\tilde{t})=-\frac{1}{2} \ln \tilde{V}_{d} \int d \tilde{\phi}\left(\tilde{t}^{\prime}\right) \sqrt{-\tilde{g}\left(\tilde{t}^{\prime}\right)} e^{-2 \tilde{\phi}\left(\tilde{t}^{\prime}\right)} \delta\left(\tilde{\phi}(\tilde{t})-\tilde{\phi}\left(\tilde{t}^{\prime}\right)\right)=d(\tilde{t})-\frac{1}{2} \ln \tilde{V}_{d}
$$

where $V_{d}=\int d^{d} x^{\prime} d^{D} \tilde{x}^{\prime}$ and $\tilde{V}_{d}=\int d^{D} x^{\prime} d^{d} \tilde{x}^{\prime}$. In the previous work [33], we have shown that the DFT action can be simplified in the cosmological background:

$$
S=-\int d t d \tilde{t} e^{-2 d}\left[\frac{1}{8} \operatorname{Tr}\left(\frac{\partial M}{\partial \tilde{t}} \frac{\partial M^{-1}}{\partial \tilde{t}}\right)+4\left(\frac{\partial d}{\partial \tilde{t}}\right)^{2}+\frac{1}{8} \operatorname{Tr}\left(\frac{\partial M}{\partial t} \frac{\partial M^{-1}}{\partial t}\right)+4\left(\frac{\partial d}{\partial t}\right)^{2}\right]
$$

\footnotetext{
${ }^{1}$ We thank C. Ma for reminding us of this.
} 
with

$$
M=\left(\begin{array}{cc}
G^{-1} & -G^{-1} B \\
B G^{-1} & G-B G^{-1} B
\end{array}\right),
$$

where $G$ and $B$ are spatial parts of $g_{i j}(t)$ and $b_{i j}(t)$. To obtain regular solutions which smoothly connect the pre- and post- big bangs, we set the dilaton potential to take a special form

$$
V(t, \tilde{t})=V_{0} e^{8 d_{*}(t, \tilde{t})} .
$$

where $V_{0}$ is a function of $V_{d}$ and $\tilde{V}_{d}$, With this non-local dilaton potential, we find

$$
\begin{aligned}
S=-\int d t d \tilde{t} e^{-2 d} & {\left[\frac{1}{8} \operatorname{Tr}\left(\frac{\partial M}{\partial \tilde{t}} \frac{\partial M^{-1}}{\partial \tilde{t}}\right)+4\left(\frac{\partial d}{\partial \tilde{t}}\right)^{2}\right.} \\
& \left.+\frac{1}{8} \operatorname{Tr}\left(\frac{\partial M}{\partial t} \frac{\partial M^{-1}}{\partial t}\right)+4\left(\frac{\partial d}{\partial t}\right)^{2}-V_{0} e^{8 d_{*}(t, \tilde{t})}\right] .
\end{aligned}
$$

To calculate the EOM of this action, the strong constraint oughts to be imposed and then all fields depend on only one temporal direction. Therefore, the dilaton and the redefined non-local dilaton coincide as $d(t)$ or $d(\tilde{t})$. Two optional actions are given as follows:

$$
S=-\int d t d \tilde{t} e^{-2 d}\left[\frac{1}{8} \operatorname{Tr}\left(\frac{\partial M}{\partial \tilde{t}} \frac{\partial M^{-1}}{\partial \tilde{t}}\right)+4\left(\frac{\partial d}{\partial \tilde{t}}\right)^{2}-V_{0} e^{8 d}\right]
$$

or

$$
S=-\int d t d \tilde{t} e^{-2 d}\left[\frac{1}{8} \operatorname{Tr}\left(\frac{\partial M}{\partial t} \frac{\partial M^{-1}}{\partial t}\right)+4\left(\frac{\partial d}{\partial t}\right)^{2}-V_{0} e^{8 d}\right] .
$$

The solutions of these two actions and their physical implications are given in refs. [32, 33].

\section{Conclusion}

In literature, it was believed that DFT only admits trivial dilaton potentials. In this paper, after presenting the gauge transformations of DFT, we introduced a non-local $O(D, D)$ invariant dilaton in the DFT formalism. We showed that this non-local dilaton is a consistent gauge invariant under the strong constraint. It is therefore possible to include loop corrections in the formalism of DFT. Our construction reduces to the ordinary dilaton when the fields depend on one single coordinate. It is of interest to extend the construction to more general backgrounds. The strong constraint is crucial in our construction. There may exist some weaker conditional results. Moreover, it is also significant to consider the dilaton potential combined with $\alpha^{\prime}$ corrections, where the gauge transformations are slightly modified.

\section{Acknowledgments}

This work is supported in part by the NSFC (Grant No. 11175039 and 11375121) and SiChuan Province Science Foundation for Youths (Grant No. 2012JQ0039). 
Open Access. This article is distributed under the terms of the Creative Commons Attribution License (CC-BY 4.0), which permits any use, distribution and reproduction in any medium, provided the original author(s) and source are credited.

\section{References}

[1] W. Siegel, Two vierbein formalism for string inspired axionic gravity, Phys. Rev. D 47 (1993) 5453 [hep-th/9302036] [INSPIRE].

[2] W. Siegel, Superspace duality in low-energy superstrings, Phys. Rev. D 48 (1993) 2826 [hep-th/9305073] [INSPIRE].

[3] W. Siegel, Manifest duality in low-energy superstrings, in Proceedings, Strings 93, Berkeley U.S.A. (1993), pg. 353 and State U. New York Stony Brook preprint ITP-SB-93-050 [hep-th/9308133] [INSPIRE].

[4] C. Hull and B. Zwiebach, Double field theory, JHEP 09 (2009) 099 [arXiv:0904.4664] [INSPIRE].

[5] C. Hull and B. Zwiebach, The gauge algebra of double field theory and Courant brackets, JHEP 09 (2009) 090 [arXiv:0908.1792] [INSPIRE].

[6] O. Hohm, C. Hull and B. Zwiebach, Background independent action for double field theory, JHEP 07 (2010) 016 [arXiv: 1003.5027] [INSPIRE].

[7] O. Hohm, C. Hull and B. Zwiebach, Generalized metric formulation of double field theory, JHEP 08 (2010) 008 [arXiv: 1006.4823] [INSPIRE].

[8] A.A. Tseytlin, Duality symmetric formulation of string world sheet dynamics, Phys. Lett. B 242 (1990) 163 [inSPIRE].

[9] A.A. Tseytlin, Duality symmetric closed string theory and interacting chiral scalars, Nucl. Phys. B 350 (1991) 395 [inSPIRE].

[10] M.J. Duff, Duality rotations in string theory, Nucl. Phys. B 335 (1990) 610 [InSPIRE].

[11] M.J. Duff and J.X. Lu, Duality rotations in membrane theory, Nucl. Phys. B 347 (1990) 394 [INSPIRE].

[12] B. Zwiebach, Double field theory, T-duality and Courant brackets, Lect. Notes Phys. 851 (2012) 265 [arXiv:1109.1782] [INSPIRE].

[13] G. Aldazabal, D. Marques and C. Núñez, Double field theory: a pedagogical review, Class. Quant. Grav. 30 (2013) 163001 [arXiv:1305.1907] [INSPIRE].

[14] D.S. Berman and D.C. Thompson, Duality symmetric string and M-theory, arXiv: 1306.2643 [INSPIRE].

[15] O. Hohm, D. Lüst and B. Zwiebach, The spacetime of double field theory: review, remarks and outlook, Fortsch. Phys. 61 (2013) 926 [arXiv:1309.2977] [INSPIRE].

[16] O. Hohm and S.K. Kwak, Massive type II in double field theory, JHEP 11 (2011) 086 [arXiv:1108.4937] [INSPIRE].

[17] G. Aldazabal, W. Baron, D. Marques and C. Núñez, The effective action of double field theory, JHEP 11 (2011) 052 [Erratum ibid. 11 (2011) 109] [arXiv:1109.0290] [INSPIRE].

[18] D. Geissbuhler, Double field theory and $N=4$ gauged supergravity, JHEP 11 (2011) 116 [arXiv:1109.4280] [INSPIRE]. 
[19] D. Geissbuhler, D. Marques, C. Núñez and V. Penas, Exploring double field theory, JHEP 06 (2013) 101 [arXiv: 1304.1472] [INSPIRE].

[20] D.S. Berman, C.D.A. Blair, E. Malek and M.J. Perry, The $O_{D, D}$ geometry of string theory, Int. J. Mod. Phys. A 29 (2014) 1450080 [arXiv:1303.6727] [InSPIRE].

[21] M. Graña and D. Marques, Gauged double field theory, JHEP 04 (2012) 020 [arXiv: 1201.2924$]$ [INSPIRE].

[22] O. Hohm and S.K. Kwak, Frame-like geometry of double field theory, J. Phys. A 44 (2011) 085404 [arXiv:1011.4101] [INSPIRE].

[23] I. Jeon, K. Lee and J.-H. Park, Stringy differential geometry, beyond Riemann, Phys. Rev. D 84 (2011) 044022 [arXiv:1105.6294] [INSPIRE].

[24] O. Hohm and B. Zwiebach, On the Riemann tensor in double field theory, JHEP 05 (2012) 126 [arXiv:1112.5296] [INSPIRE].

[25] O. Hohm and B. Zwiebach, Towards an invariant geometry of double field theory, J. Math. Phys. 54 (2013) 032303 [arXiv:1212.1736] [inSPIRE].

[26] M. Gasperini, Elements of string cosmology, Cambridge Univ. Pr., Cambridge U.K. (2007) [INSPIRE].

[27] O. Hohm, W. Siegel and B. Zwiebach, Doubled $\alpha^{\prime}$-geometry, JHEP 02 (2014) 065 [arXiv:1306.2970] [INSPIRE].

[28] O. Hohm and B. Zwiebach, Green-Schwarz mechanism and $\alpha^{\prime}$-deformed Courant brackets, arXiv: 1407.0708 [INSPIRE].

[29] O. Hohm and B. Zwiebach, Double field theory at order $\alpha^{\prime}$, arXiv:1407.3803 [INSPIRE].

[30] O.A. Bedoya, D. Marques and C. Núñez, Heterotic $\alpha^{\prime}$-corrections in double field theory, arXiv: 1407.0365 [INSPIRE].

[31] M. Gasperini, M. Giovannini and G. Veneziano, Perturbations in a nonsingular bouncing universe, Phys. Lett. B 569 (2003) 113 [hep-th/0306113] [INSPIRE].

[32] H. Wu and H. Yang, Double field theory inspired cosmology, JCAP 07 (2014) 024 [arXiv: 1307.0159] [INSPIRE].

[33] H. Wu and H. Yang, New cosmological signatures from double field theory, arXiv:1312.5580 [INSPIRE].

[34] C.-T. Ma and C.-M. Shen, Cosmological implications from $O_{D, D}$, arXiv:1405.4073 [INSPIRE].

[35] I. Jeon, K. Lee and J.-H. Park, Differential geometry with a projection: application to double field theory, JHEP 04 (2011) 014 [arXiv: 1011.1324] [INSPIRE].

[36] I. Jeon, K. Lee and J.-H. Park, Double field formulation of Yang-Mills theory, Phys. Lett. B 701 (2011) 260 [arXiv: 1102.0419] [InSPIRE].

[37] J.-H. Park, Comments on double field theory and diffeomorphisms, JHEP 06 (2013) 098 [arXiv: 1304.5946] [INSPIRE].

[38] O. Hohm and B. Zwiebach, Large gauge transformations in double field theory, JHEP 02 (2013) 075 [arXiv: 1207.4198] [INSPIRE]. 